\title{
Effect of Simvastatin on Proliferation of Vascular Smooth Muscle Cells During Delayed Cerebral Vasospasm After Subarachnoid Hemorrhage
}

\author{
Hongzhou DUAN, Jiayong ZHANG, Liang LI, Shengde BAO \\ Peking University First Hospital, Department of Neurosurgery, Beijing, China
}

\section{ABSTRACT}

AIM: To explore the effect of simvastatin on proliferation of vascular smooth muscle cells (VSMCs) during delayed cerebral vasospasm (dCVS) after subarachnoid hemorrhage (SAH).

MATERIAL and METHODS: Thirty-six male New Zealand White rabbits were randomly divided into three groups: 1) Control group $(n=12)$ : given conventional breeding and normal sodium (0.9\%) was injected twice into the cisterna magna. 2) SAH group ( $n=12)$ : given conventional breeding and a SAH model was established. 3) Simvastatin + SAH group ( $n=12)$ : given conventional breeding and simvastatin for one week, and then a SAH model was established. The first cerebral angiography was conducted before the first injection of sodium or autologous blood into the cisterna magna. The second angiography was done three days after the second injection. The ultrastructural pathology of the basilar artery was compared in three groups. The expression of platelet-derived growth factor- $\beta$ (PDGF- $\beta$ ), proliferating cell nuclear antigen (PCNA) and a-smooth muscle actin (a-SMA) in VSMCs was analysed by RT-PCR.

RESULTS: Angiography examinations showed that the basilar artery was obviously contracted in the SAH group and dCVS was confirmed existence after blood injection into the cisterna magna twice. The thickness of VSMCs in the SAH group increased and the expression of PDGF- $\beta$, PCNA, and $a-S M A$ in SAH group were all increased compared to the control group ( $p<0.05$ ), and decreased while prophylactic giving simvastatin $(p<0.05)$.

CONCLUSION: Simvastatin may relieve dCVS after SAH by inhibiting the proliferation of VSMCs.

KEYWORDS: Subarachnoid hemorrhage, Delayed cerebral vasospasm, Vascular smooth muscle cell, Proliferation

\section{INTRODUCTION}

Stroke is one of the leading causes of death and the main reason for long-term disability. Five percent patients with stroke are caused by subarachnoid hemorrhage (SAH) (13). Delayed cerebral vasospasm (dCVS) is a common and dangerous complication of SAH. It usually occurs within 4-15 days after $\mathrm{SAH}$ and results in serious and irreversible delayed cerebral injury (DCl). dCVS is the main cause of morbidity and mortality after SAH (1). But the pathogenesis of dCVS is still unclear.
Recently, many studies reported that it may be related with the proliferation of vascular smooth muscle cells (VSMCs) $(5,9)$.

Platelet-derived growth factor- $\beta$ (PDGF- $\beta$ ) is a polypeptide which could stimulate vascular smooth muscle cell and matrix proliferation (14). Proliferating cell nuclear antigen (PCNA) is a marker of deoxyribonucleic acid (DNA) synthesis, and it can be considered as an index in evaluating cell proliferation (20). a-smooth muscle actin (a-SMA) is an important functional protein in VSMCs, and the change of its expression could 
reveal the change of phenotype and function of VSMCs (2). Simvastatin, as one kind of statins, has some other effects besides reducing cholesterol, such as inhibiting the proliferation and migration of smooth muscle cells, improving vessel endothelium function, inhibiting inflammation and oxidative stress, inhibiting platelet function, thrombosis and formation of new vessels (10). Recently, some researches discovered that simvastatin could prevent dCVS after SAH, but the mechanisms of prevention are still unclear $(6,12)$.

In this research, we will explore the effect of simvastatin on the expression of PDGF- $\beta$, PCNA and a-SMA in VSMCs during dCVS after $\mathrm{SAH}$, and illuminate the mechanism of simvastatin in preventing dCVS.

\section{MATERIAL and METHODS}

\section{Animals and Groups}

Thirty-six adult male New Zealand White rabbits $(2.5-3.5 \mathrm{~kg})$ were bought from Department of Laboratory Animal Science, Peking University First Hospital. They were divided into three groups equally: 1) Control group ( $n=12)$ : being given conventional breeding for one week, and then the first cerebral angiography was conducted. Normal saline $(0.9 \%, 1 \mathrm{ml} / \mathrm{kg})$ was injected into the cisterna magna. Forty-eight hours later, same dose of normal saline was injected in same way. The second cerebral angiography was conducted three days later to observe the change of diameter in basilar artery. 2) SAH group $(n=12)$ : all steps were the same as control group except that the fluid injected into the cisterna magna was arterial blood (1 ml/kg) without anticoagulant. 3) Simvastatin $+\mathrm{SAH}$ group ( $\mathrm{n}=12)$ : Simvastatin $(5 \mathrm{mg} / \mathrm{kg} / \mathrm{d})$ was administered by intragastic way for one week, and other processes were the same as SAH group. After second angiography, all rabbits were sacrificed. The basilar artery was taken out, one part for electron microscope examination, and others were reserved in $-70^{\circ} \mathrm{C}$ refrigerator.

\section{SAH Model}

Rabbits were anesthetized with pentobarbital $(250 \mathrm{mg} /$ $\mathrm{kg}$ i.v.). After sterilization, trochar (Type 12) was punctured vertically from the skin $0.5 \mathrm{~cm}$ below occipital tuberosity until there was a breakthrough feeling and cerebrospinal fluid flew out. Autologous blood from the auricular artery without anticoagulant $(1 \mathrm{ml} / \mathrm{kg})$ was injected into the subarachnoid space slowly. Then rabbit was placed in the head-down position for 30 minutes to make injected blood adhere to basilar artery. Forty-eight hours later, the same procedure was repeated.

\section{Rabbit Cerebral Angiography}

After general anesthesia, the right common femoral artery was exposed and a $4 \mathrm{~F}$ catheter was inserted and sent to left subclavian artery under roadmap, then another microcatheter was placed into V2 section of left vertebral artery. Omnipaque was injected and the image of vertebrobasilar artery was acquired. The diameter of basilar artery was measured directly. After pulling out the catheters, the femoral artery was sutured.

\section{Perfusion and Harvest Basilar Artery}

Three days after the second angiography, thoracotomy was performed after general anesthesia, and perfusion fluid (glutaraldehyde, 2.5\%) was irrigated into the left ventricle. When effluent fluid from right auricle was clear, craniotomy was performed and the basilar artery was taken out, one part of basilar artery was fixed for electron microscope, others were kept in liquid nitrogen for pathological examination.

\section{Electron Microscope Examination}

Basilar artery was fixed into glutaraldehyde (3\%) and osmic acid $(1 \%)$ in turn, and then be dehydrated in acetone and Epon812 for embedding. Ultrathin section (LEICA UCT, Germany) was double stained by uranyl acetate and citric acid. Result was observed under transmission electron microscope (JEM1230, Japan).

\section{Immunofluorescence}

The protocol was similar to immunohistochemistry. Primary antibody of a-SMA (mouse anti rabbit, 1:400, Boshi De Company, Wuhan, China) and FITC labeled fluorescence secondary antibody (goat anti mouse, 1:100, Zhongshan Company, Beijing, China) were used. Results were observed under confocal fluorescence microscope and were compared according to the intensity of cell's fluorescence: Define no fluorescence light as (-), extremely weak light as $( \pm)$, weak light but clear to observe as (+), bright light as (++), blink light as $(+++)$.

\section{Immunohistochemistry Examination}

Immunohistochemistry examination was procedured following the specification. Primary antibody of PCNA (mouse anti rabbit, 1:150, Boshi De Company, Wuhan, China) and secondary antibody (goat anti mouse, 1:200, Zhongshan Company, Beijing, China) were used. The results were observed under microscope. Five high power vision (x400) were selected randomly including 500 cells, and the percent of positive cells were calculated.

\section{RNA Extraction and Real-time PCR}

Total RNA was extracted from basilar artery with Trizol reagent (Huamei Company, Beijing, China) following the instruction. Use following reaction system for reverse transcription: Buffer $5 \mathrm{ul}, 2.5 \mathrm{mM}$ dNTP $5 \mathrm{ul}, \mathrm{MMLV}-\mathrm{RT} 1 \mathrm{ul}, 42^{\circ} \mathrm{C} 60 \mathrm{~min}$. PDGF- $\beta$ cDNA was cloned by RT-PCR. SYBR Green Real-Time PCR was carried out to quantify the expression. The reaction system (50 ul) was as follows: $2^{*}$ SyBR Green Buffer 25 ul, primer $3 \mathrm{ul}, \mathrm{cDNA} 3 \mathrm{ul}$, and add $\mathrm{H}_{2} \mathrm{O}$ to $50 \mathrm{ul}$. The procedure was: denaturation at $95^{\circ} \mathrm{C}$ for $10 \mathrm{~min}$, then 40 cycles at $95^{\circ} \mathrm{C} 15 \mathrm{~s}$, and extension at $60^{\circ} \mathrm{C}$ for one minute. Sense primer sequence of PDGF- $\beta$ was 5'-CCCATCTACATCATCACCGAGTAC-3', while antisense primer sequence was 5'-GAGTGCTGCTGCAGGAAGGT-3'. Sense primer sequence of GAPDH was 5'-CCATCACCATCTTCCAGGAG-3', and antisense primer sequence was 5'-CCAGCTTCACCA CCTTCTTG-3'. A series of purified multiproportion diluted PCR production were set as standard substance. The standard substance, samples and negative control (deionized water) were amplified together each time 
and standard solubility curve of GAPDH and PDGF- $\beta$ were obtained. Based on this curve the relative concentration of GAPDH and PDGF- $\beta$ in the sample could be calculated. The ratio of PDGF- $\beta / G A P D H$ represented the relative expression of PDGF- $\beta$ in sample. Each cDNA sample should be repeated for 2-3 tubes, and the average ratio was calculated as the result.

\section{Statistical Analysis}

Results were expressed as mean \pm standard deviation. Continuous variables were compared by analysis of variance. Ranked data was compared by rank sum test. All data were analyzed by SPSS version 10.0 for Windows (SPSS Inc, Chicago, IL). $p$ value $<0.05$ was considered as statistically significant.

\section{- RESULTS}

\section{Cerebral Angiography}

All rabbits finished the experiment successfully except one in control group, two in SAH group and one in Simvastatin+SAH group died because of puncturing into the brain stem. There was no obvious change in the diameter of basilar artery in control group $(0.87 \pm 0.06 \mathrm{~mm}$, Figure $1 \mathrm{~A})$, while the basilar arteries in SAH group contracted, parts of which contracted like beads on string, even in some cases, the distal end couldn't be observed $(0.68 \pm 0.09 \mathrm{~mm}$, Figure $1 \mathrm{~B})$. In simvastatin+SAH group, the basilar artery also contracted after the second injection of autologous blood $(0.77 \pm 0.08 \mathrm{~mm}$, Figure $1 \mathrm{C})$, but the diameter of basilar artery was larger than $\mathrm{SAH}$ group $(0.77 \pm 0.08 \mathrm{~mm}$ vs $0.68 \pm 0.09 \mathrm{~mm}, \mathrm{p}<0.05)$.

\section{Morphological Examination}

Hematoxylin and eosin (H\&E) staining: The endomembrane of rabbit basilar artery in control group was smooth, and smooth muscle was thin $(44.37 \pm 8.65 \mu \mathrm{m}$, Figure $2 \mathrm{~A})$. In $\mathrm{SAH}$ group, the smooth muscle was a little thicker $(76.26 \pm 12.73 \mu \mathrm{m}$, Figure 2B). In simvastatin+SAH group, the thickness decreased a little $(61.82 \pm 16.95 \mu \mathrm{m}$, Figure $2 \mathrm{C})$.

Electron microscope: Endothelial cells in control group were thin and flat, VSMCs were spindle-shaped with many microfilaments and little cell organelles surrounding nucleus (Figure 3A). In SAH group, the endothelial cells augmented in shape with more chondriosome vacuoles. VSMCs were also enlarged with abundant cell organelles and extended vertically to endomembrane, a lot of lamellar body and vacuoles existed in cytoplasm (Figure 3B). In simvastatin+SAH group, chondriosome in endothelial cells was less swollen and vacuoles were sporadic, VSMCs returned to fusiform shape, and lamellar body was observed infrequently in cytoplasm (Figure 3C).
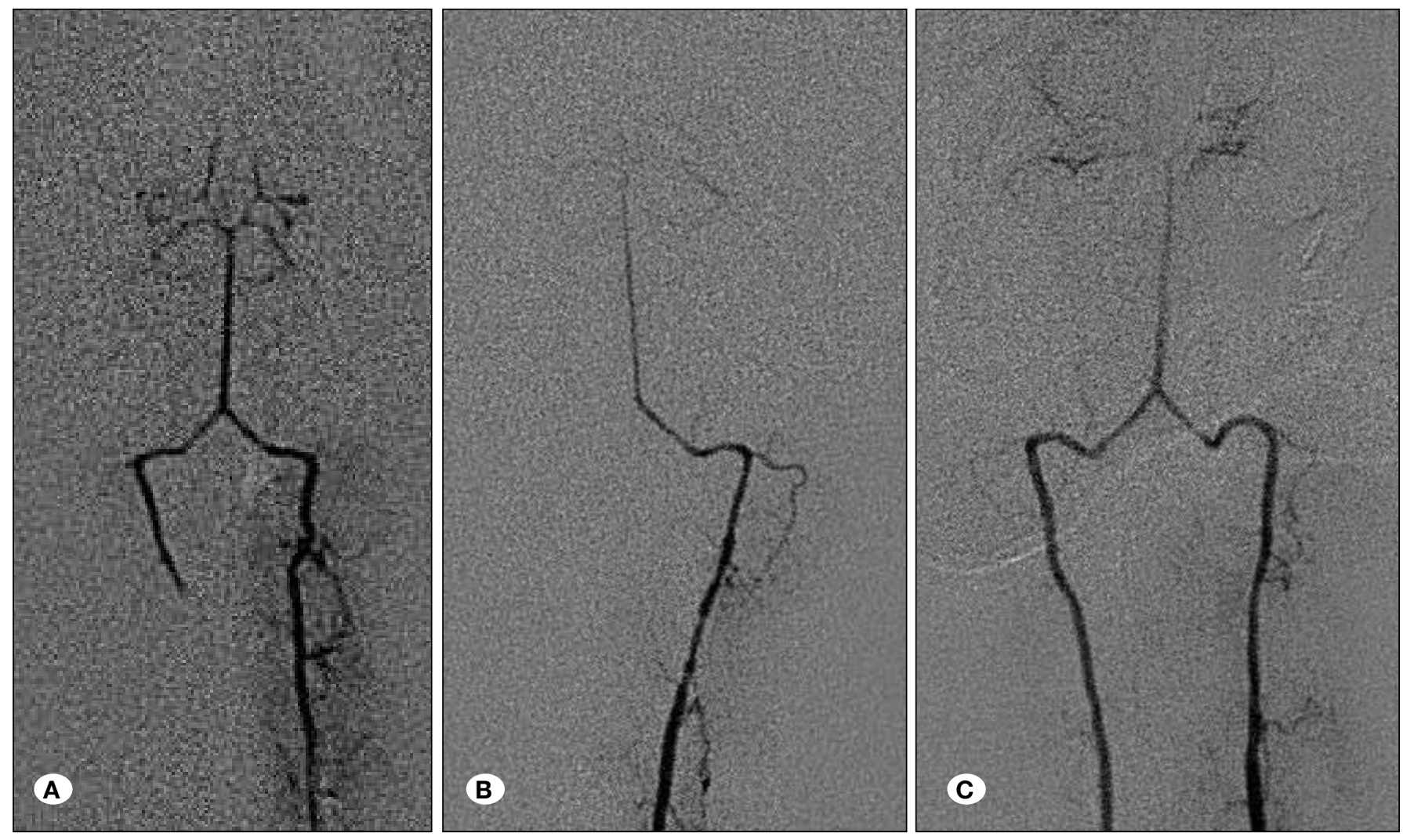

Figure 1: A) Vertebral angiography in control group showed normal vertebral artery and basilar artery. B) Vertebral angiography in SAH group showed vertebrobasilar artery contracted like beads on string, the distal end couldn't be observed. C) Vertebral angiography in Simvastatin+SAH group showed contracted artery relieved. 


\section{a-SMA Expression}

In control group, the fluorescence intensity of a-SMA in VSMCs was light (Figure 4A), while it increased significantly in $\mathrm{SAH}$ group (Figure 4B, $\mathrm{p}<0.05$ ). After adding simvastatin, the intensity decreased (vs $\mathrm{SAH}$ group) (Figure $4 \mathrm{C}, \mathrm{p}<0.05$ ) (Table I).

\section{PCNA Expression}

The positive PCNA expression in a cell was brown staining in nucleus. In SAH group (Figure 5B), the expression of PCNA in VSMCs was higher than in control group (Figure 5A). While in simvastatin group, the expression of PCNA was reduced (Figure 5C, p<0.05) (Table II).

\section{PDGF- $\beta$ Expression}

Real-Time PCR examination showed that the expression of PDGF- $\beta$ gene in SAH group was higher than control group $(p<0.05)$. While in simvastatin $+\mathrm{SAH}$ group, the intensity of PDGF- $\beta$ gene expression reduced compared with $\mathrm{SAH}$ group $(p<0.05)$ (Table III).

\section{DISCUSSION}

More than half of $\mathrm{SAH}$ patients develop angiographically detectable dCVS. It mostly occurs between days 4 and 15 after ictus and can be associated with neurological deficits that contribute to increased morbidity and mortality after SAH (8). Although dCVS is well studied, the exact mechanism still hasn't been illustrated. The proliferation and phenotype change of VSMC may be one of the pathogenesis of dCVS after $\mathrm{SAH}(6)$.

PCNA and smooth muscle actin are two biomarkers related to proliferation of VSMC. PCNA is synthetized and resides in cell nucleus. Actin is the elemental component of microfilament. a-actin expressed in vascular smooth muscle as a-SM-actin (a-SMA) is an important functional protein in VSMCs, and its change in expression plays an important role in curing the structural and functional change of VSMCs (21). The expression of PCNA and a-SMA increased in experimental $\mathrm{SAH}$ models $(5,17)$. Rosiglitazone and a mitogen-activated protein kinase inhibitor (PD98059) can improve vasospasm
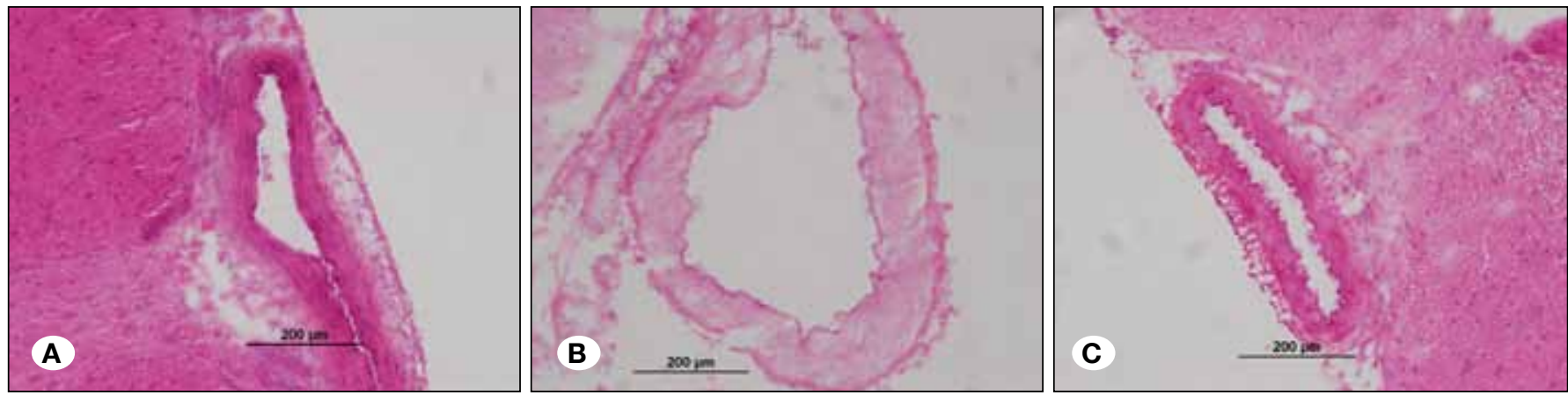

Figure 2: A) H\&E staining of control group ( $\times 200)$ showed the endomembrane of basilar artery was smooth, and smooth muscle was thin. B) H\&E staining of SAH group $(\times 200)$ showed the smooth muscle was thick. C) H\&E staining of Simvastatin + SAH group $(\times 200)$ showed the thickness of smooth muscle decreased.
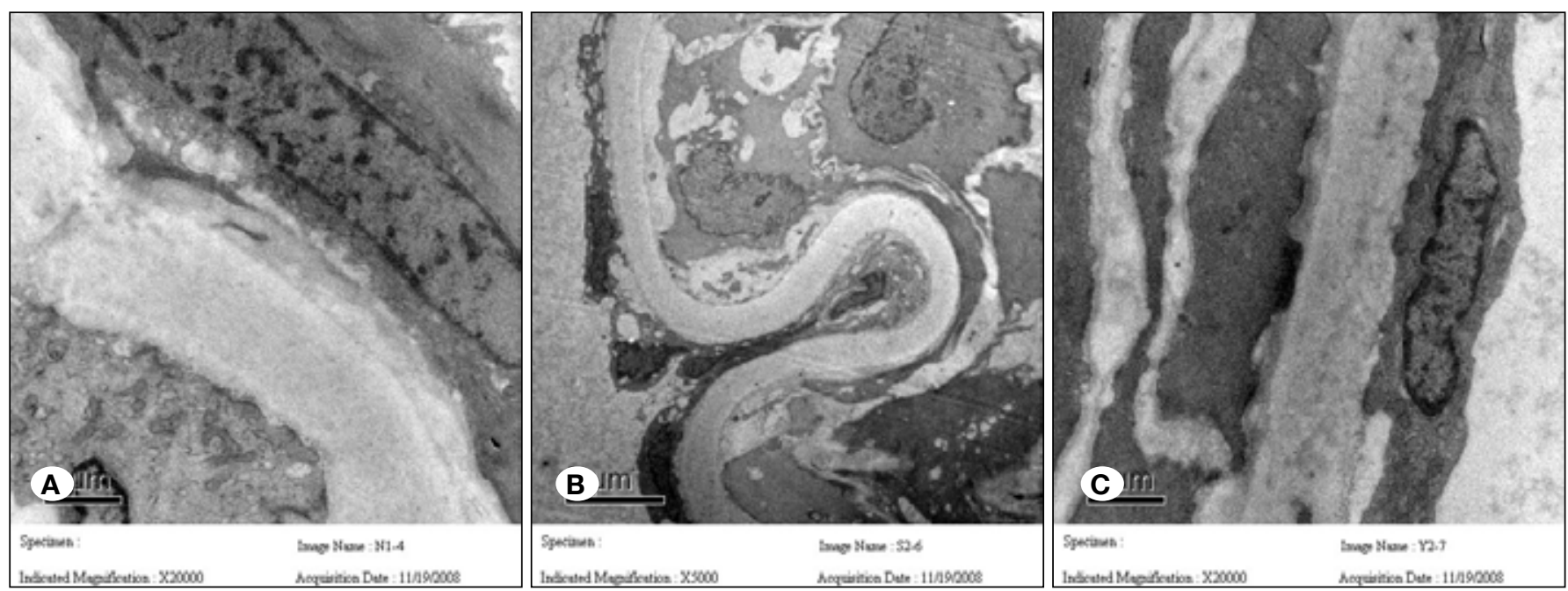

Figure 3: A) Electron microscopy (×20000) showed endothelial cells and VSMCs in control group were normal. B) In SAH group ( $\times 5000)$, the endothelial cells and VSMCs were enlarged with abundant cell organelles, VSMCs extended vertically to endomembrane with a lot of lamellar body and vacuoles in cytoplasm. C) In Simvastatin+SAH group (×20000), chondriosome in endothelial cells was less swollen, VSMCs returned to fusiform shape with less lamellar body in cytoplasm. 
Duan H. et al: Simvastatin in Cerebral Vasospasm

Table I: The Expression of a-SMA

\begin{tabular}{|c|c|c|c|c|c|c|}
\hline Group & \multicolumn{5}{|c|}{ Fluorescence intensity (amount) } & Mean Rank \\
\hline Control $(n=11)$ & 1 & 6 & 4 & 0 & 0 & 8.27 \\
\hline $\mathrm{SAH}(n=10)$ & 0 & 0 & 1 & 2 & 7 & $26.50^{*}$ \\
\hline $\mathrm{SAH}+$ Simvastatin $(\mathrm{n}=11)$ & 0 & 1 & 8 & 2 & 0 & $15.64^{\#}$ \\
\hline
\end{tabular}

*: $p<0.05$ stands for compared with control group, \#: $P<0.05$ stands for compared with SAH group.

Table II: The Expression of PCNA

\begin{tabular}{|c|c|c|c|c|c|}
\hline \multirow{2}{*}{ Group } & \multicolumn{4}{|c|}{ Staining intensity (amount) } & \multirow{2}{*}{ Mean Rank } \\
\hline & - & + & ++ & +++ & \\
\hline Control $(n=11)$ & 3 & 7 & 1 & 0 & 9.18 \\
\hline SAH $(n=10)$ & 0 & 0 & 3 & 7 & $25.60^{\star}$ \\
\hline $\mathrm{SAH}+$ Simvastatin $(\mathrm{n}=11)$ & 1 & 5 & 3 & 2 & $15.55^{\#}$ \\
\hline
\end{tabular}

*: $p<0.05$ stands for compared with control group, \#: $P<0.05$ stands for compared with SAH group.
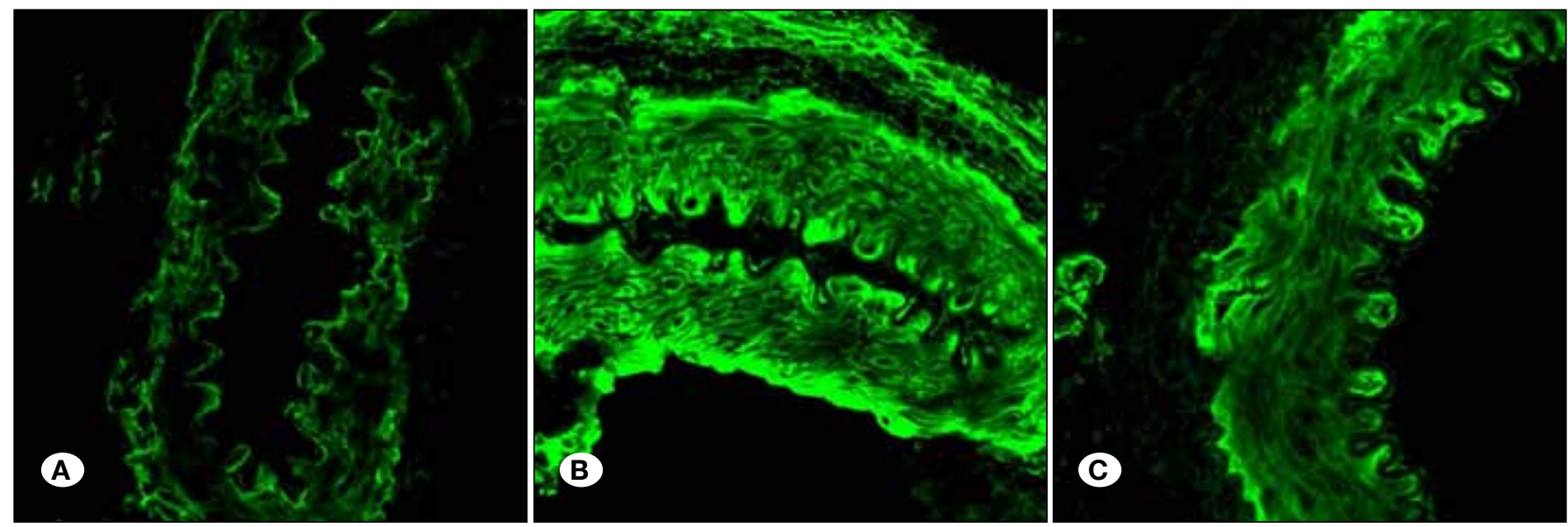

Figure 4: A) In control group ( $\times 600)$, the fluorescence intensity of a-SMA in VSMCs was light. B) In SAH group ( $\times 600)$, the fluorescence intensity of a-SMA increased significantly. C) In Simvastatin+SAH group (x600), the fluorescence intensity of a-SMA decreased compared with SAH group.
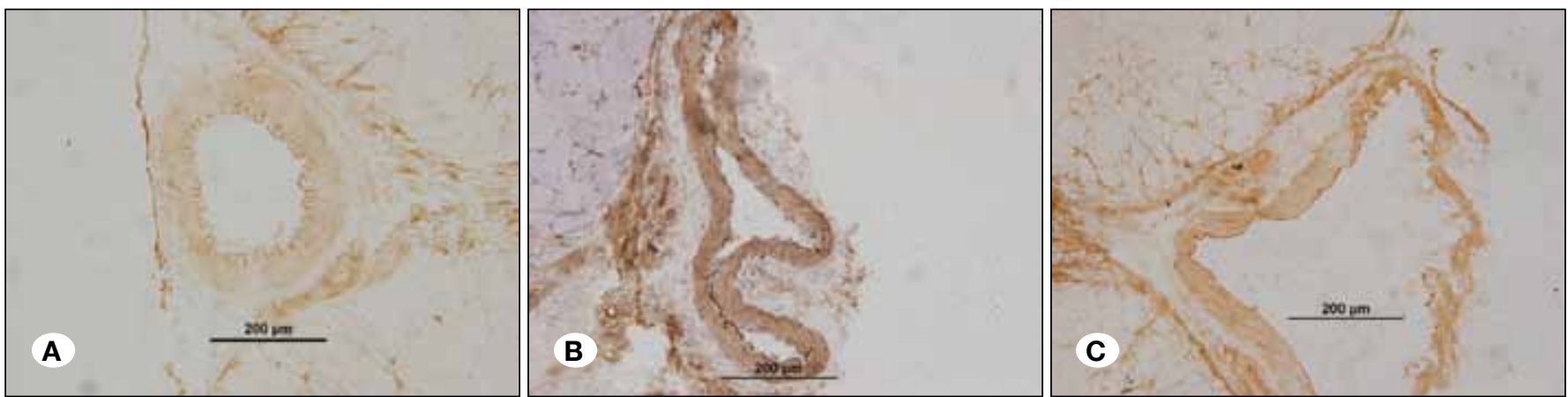

Figure 5: A) In control group (×200), PCNA expression is light. B) In SAH group (×200), the expression of PCNA in VSMCs was higher. C) In Simvastatin+SAH group ( $\times 200)$, the expression of PCNA reduced. 
Table III: The Expression of PDGF- $\beta$

\begin{tabular}{lc}
\hline Group & PDGF- $\beta /$ GAPDH \\
\hline Control $(n=11)$ & $0.670 \pm 0.121$ \\
\hline SAH $(n=10)$ & $0.845 \pm 0.287^{*}$ \\
\hline SAH + Simvastatin $(n=11)$ & $0.766 \pm 0.238^{\#}$ \\
\hline F & 161.377 \\
\hline
\end{tabular}

*: $p<0.05$ stands for compared with control group, \#: $P<0.05$ stands for compared with SAH group.

by decrease the expression of PCNA and a-SMA through ERK1/2 phosphorylation signaling pathway and Akt signaling pathway $(3,17)$.

PDGF is a polypeptide generated by many kinds of cells, which could stimulate the proliferation of VSMC, colloid, and so on. It is a homodimer or heterodimer which is generated by conjunction of $a$ and $\beta$ polypeptide chain. The bioactive PDGF- $\beta$ could stimulate the proliferation of VSMC and fibroblast cells. It also has chemotaxis to neutrophils, SMCs and fibroblasts and induces the contraction of blood vessel. A rabbit model of CVS demonstrated that PDGF- $\beta$ expression was observed in VSMC as early as $3 \mathrm{~h}$ post-SAH and was evident after 1 day and reached a peak in 7 days, suggesting that PDGF- $\beta$ may take part in the pathogenesis in dCVS and may be a target of treatment (7). Shiba et al. confirmed PDGF playing an important role in the pathogenesis of vasospasm after SAH and found imatinib significantly inhibited post-SAH PDGFR activation and prevented vasospasm significantly (16). The inhibiting effect of imatinib may be via inhibiting tenascin- $C$ expression (15).

Simvastatin is an inhibitor of HMG-CoA reductase. In clinical practice, it mainly acts as a drug adjusting hyperlipemia. Recently, more researches on simvastatin indicated that it had many other effects, including inhibiting proliferation and movement of VSMCs, improving the function of endothelium, inhibiting inflammation, oxidative stress, platelet function, thrombosis and formation of new vessels (22). Lynch et al. (11) reported a randomized clinical trial which demonstrated that the use of simvastatin as prophylaxis against delayed cerebral ischemia after aneurysmal SAH was a safe and well-tolerated intervention, its use attenuates serum markers associated with brain injury and decreased the incidence of radiographic vasospasm and delayed ischemic deficit. Further randomized trials conducted by Tseng et al. $(18,19)$ confirmed that acute statin treatment reduced traditional rescue therapy for vasospasm after aneurysmal SAH. It not only improved mortality related to vasospasm, but also improved the physical and psychosocial outcome of 6 months. Other researchers reported the same phenomenon $(6,12)$, but the mechanism has not been comprehensively illustrated till now.

In this research, we established the rabbit SAH model through injecting autologous blood twice into the cisterna magna and confirmed the existence of CVS by angiography. Morphological examinations showed proliferation in vessel's wall and VSMCs during dCVS. We also detected the expression of
PDGF- $\beta$, PCNA and $\alpha-S M A$ in basilar artery and found that the expression of these three markers in SAH group were significantly higher than in control group and attenuated after adding simvastatin. These results above may indicate that proliferation of VSMCs may play an important role during dCVS after $\mathrm{SAH}$, and simvastatin may improve the outcome of dCVS by attenuating the proliferation of VSMCs.

There are two phenotypes of VSMCs as, contracted type and synthetized type, respectively. When the expression of a-SMA (marker of myofibroblast) increases, it means that VSMCs transform from synthesized type to contracted type, vice versa. In this research, the positive expression of a-SMA in VSMCs in $\mathrm{SAH}$ group was significantly more than control group. This indicates that VSMCs transformed from synthesized type to contracted type when dCVS happened after SAH. The abundant contracted VSMCs could induce the contraction of the vessel and finally led to the formation of dCVS. After prophylactic giving simvastatin, the expression of a-SMA in VSMCs decreased compared with SAH group, which might indicate that simvastatin may inhibit the transformation of VSMCs from synthesized type to contracted type, this might be another possible reason why simvastatin partially relieve dCVS.

Recent research showed that paeonol inhibited proliferation of VSMCs stimulated by high glucose. The inhibition of PDGF- $\beta$ secretion was through Ras-Raf-ERK1/2 signaling pathway in VSMCs (4). Whether simvastatin inhibiting the secretion of PDGF- $\beta$ and the proliferation of VSCMs is also through RasRaf-ERK1/2 signaling pathway still needs further researches.

There are also some shortcomings in our research, such as all the results of the markers are semi-quantitative. The results we get from the rabbit model might be different from human body. To illustrate the whole efficiency, pathogenesis and signaling pathway of simvastatin in preventing dCVS, there's still a long way to go.

\section{- CONCLUSION}

Simvastatin can relieve dCVS in rabbits after $\mathrm{SAH}$, which might be related to the inhibition effect on proliferation of VSMCs. Further research may focus on the signaling pathway of this effect.

\section{- REFERENCES}

1. Baggott CD, Aagaard-Kienitz B: Cerebral vasospasm. Neurosurg Clin N Am 25: 497-528, 2014

2. Chaterji S, Lam CH, Ho DS, Proske DC, Baker AB: Syndecan-1 regulates vascular smooth muscle cell phenotype. PLoS One 9:e89824, 2014

3. Chen D, Chen JJ, Yin Q, Guan JH, Liu YH: Role of ERK1/2 and vascular cell proliferation in cerebral vasospasm after experimental subarachnoid hemorrhage. Acta Neurochir (Wien) 151:1127-1134, 2009

4. Chen J, Dai M, Wang Y: Paeonol inhibits proliferation of vascular smooth muscle cells stimulated by high glucose via Ras-Raf-ERK1/2 signaling pathway in coculture model. Evid Based Complement Alternat Med 2014:484269, 2014 
5. Cheng MF, Song JN, Li DD, Zhao YL, An JY, Sun P, Luo XH: The role of rosiglitazone in the proliferation of vascular smooth muscle cells after experimental subarachnoid hemorrhage. Acta Neurochir (Wien) 156:2103-2109, 2014

6. Chou SH, Smith EE, Badjatia N, Nogueira RG, Sims JR 2nd, Ogilvy CS, Rordorf GA, Ayata C: A randomized, double-blind, placebo-controlled pilot study of simvastatin in aneurysmal subarachnoid hemorrhage. Stroke 39:2891-2893, 2008

7. Cui HK, Yan RF, Ding XL, Zhao P, Wu QW, Wang HP, Qin HX, Tu JF, Yang RM: Platelet-derived growth factor- $\beta$ expression in rabbit models of cerebral vasospasm following subarachnoid hemorrhage. Mol Med Rep 10:1416-1422, 2014

8. Danura H, Schatlo B, Marbacher S, Kerkeni H, Diepers $\mathrm{M}$, Remonda L, Fathi AR, Fandino J: Acute angiographic vasospasm and the incidence of delayed cerebral vasospasm: Preliminary results. Acta Neurochir Suppl 120:187-190, 2015

9. Kolias AG, Sen J, Belli A: Pathogenesis of cerebral vasospasm following aneurysmal subarachnoid hemorrhage: Putative mechanisms and novel approaches. J Neurosci Res 87:1-11, 2009

10. Kolovou GD, Katerina A, loannis V, Cokkinos DV: Simvastatin: Two decades in a circle. Cardiovasc Ther 26:166-178, 2008

11. Lynch JR1, Wang $H$, McGirt MJ, Floyd J, Friedman $A H$, Coon AL, Blessing R, Alexander MJ, Graffagnino C, Warner DS, Laskowitz DT: Simvastatin reduces vasospasm after aneurysmal subarachnoid hemorrhage: Results of a pilot randomized clinical trial. Stroke 36:2024-2026, 2005

12. McGirt MJ, Pradilla G, Legnani FG, Thai QA, Recinos PF, Tamargo RJ, Clatterbuck RE: Systemic administration of simvastatin after the onset of experimental subarachnoid hemorrhage attenuates cerebral vasospasm. Neurosurgery 58:945-951, 2006

13. Sakowitz OW, Unterberg AW: Detecting and treating microvascular ischemia after subarachnoid hemorrhage. Curr Opin Crit Care 12:103-111, 2006

14. Salabei JK, Hill BG: Mitochondrial fission induced by plateletderived growth factor regulates vascular smooth muscle cel bioenergetics and cell proliferation. Redox Biol 1:542-551, 2013
15. Shiba M, Suzuki H, Fujimoto M, Shimojo N, Imanaka-Yoshida K, Yoshida T, Kanamaru K, Matsushima S, Taki W: Imatinib mesylate prevents cerebral vasospasm after subarachnoid hemorrhage via inhibiting tenascin- $\mathrm{C}$ expression in rats. Neurobiol Dis 46:172-179, 2012

16. Shiba M, Suzuki H, Fujimoto M, Shimojo N, Imanaka-Yoshida K, Yoshida T, Kanamaru K, Matsushima S, Taki W: Role of platelet-derived growth factor in cerebral vasospasm after subarachnoid hemorrhage in rats. Acta Neurochir Suppl 115:219-223, 2013

17. Song JN, An JY, Hao GS, Li DD, Sun P, Li Y, Xue JG: Role of Akt signaling pathway in delayed cerebral vasospasm after subarachnoid hemorrhage in rats. Acta Neurochir (Wien) 155:2063-2070, 2013

18. Tseng MY, Hutchinson PJ, Czosnyka M, Richards H, Pickard JD, Kirkpatrick PJ: Effects of acute pravastatin treatment on intensity of rescue therapy, length of inpatient stay, and 6-month outcome in patients after aneurysmal subarachnoid hemorrhage. Stroke 38:1545-1550, 2007

19. Tseng MY, Hutchinson PJ, Turner CL, Czosnyka M, Richards H, Pickard JD, Kirkpatrick PJ: Biological effects of acute pravastatin treatment in patients after aneurysmal subarachnoid hemorrhage: A double-blind, placebocontrolled trial. J Neurosurg 107:1092-1100, 2007

20. von Bohlen und Halbach O: Immunohistological markers for proliferative events, gliogenesis, and neurogenesis within the adult hippocampus. Cell Tissue Res 345:1-19, 2011

21. Wang J, Zohar R, McCulloch CA: Multiple roles of alphasmooth muscle actin in mechanotransduction. Exp Cell Res 312:205-214, 2006

22. Yoon SJ, Yoon YW, Lee BK, Kwon HM, Hwang KC, Kim M, Chang W, Hong BK, Lee YH, Park SJ, Min PK, Rim SJ: Potential role of HMG CoA reductase inhibitor on oxidative stress induced by advanced glycation endproducts in vascular smooth muscle cells of diabetic vasculopathy. Exp Mol Med $41: 802-811,2009$ 